\title{
Correction to: Cell-Free Biosensors and AI Integration
}

\section{Paul Soudier, Léon Faure, Manish Kushwaha, and Jean-Loup Faulon}

\section{Correction to:}

Chapter 19 in: Ashty S. Karim and Michael C. Jewett (eds.), Cell-Free

Gene Expression: Methods and Protocols, Methods in Molecular Biology, vol. 2433, https://doi.org/10.1007/978-1-0716-1998-8_19

The chapter authors name was incorrectly published and it has been updated as "Manish Kushwaha" now. 